\title{
Contributing Factors to the Clinical and Economic Burden of Patients with Laboratory-Confirmed Carbapenem-Nonsusceptible Gram-Negative Respiratory Infections
}

This article was published in the following Dove Press journal:

Infection and Drug Resistance

\author{
Eilish McCann (D) \\ Anita H Sung' \\ Gang Ye (D) ${ }^{2}$ \\ Latha Vankeepuram ${ }^{2}$ \\ Ying $P$ Tabak ${ }^{2}$ \\ 'Center for Observational and Real- \\ World Evidence (CORE), Merck \& Co. \\ Inc., Kenilworth, NJ, USA; ${ }^{2}$ Digital Health, \\ Medical Affairs, Becton, Dickinson and \\ Company, Franklin Lakes, NJ, USA
}

Correspondence: Eilish McCann Center for Observational and Real-World Evidence (CORE), Merck \& Co., Inc., 2000 Galloping Hill Road, Kenilworth, NJ 07033, USA

Tel +I 7325947472

Email eilish.mccann@merck.com

\begin{abstract}
Purpose: This study examined patient- and hospital-level predictor variables that contribute to worse clinical and economic outcomes in patients with carbapenem-nonsusceptible respiratory infections.

Patients and Methods: Electronic data (January 2013 to September 2015) were from 78 US hospitals. Nonduplicate, gram-negative respiratory isolates were considered carbapenemnonsusceptible if they tested resistant/intermediate to imipenem, meropenem, doripenem, or ertapenem. Potential predictors of outcomes (in-hospital mortality, 30-day readmission, length of stay [LOS], hospital total cost, and net gain/loss per patient) were examined using univariate analysis and generalized linear mixed models. Statistical significance and model goodness-of-fit criteria were used to identify significant predictors.
\end{abstract}

Results: A total of 1488 carbapenem-nonsusceptible respiratory patients were identified. Overall, the mortality rate was $13.7 \%$, 30-day readmission rate was $20.6 \%$, mean LOS was 20 days, mean total cost was $\$ 54,158$, and mean net loss was $\$ 139$ per patient. Our models showed that hospital-onset infection, higher clinical severity, mechanical ventilation/intensive care unit status, polymicrobial infection, and underlying diseases were all significant predictors for mortality, LOS, and total cost. Hospital-onset infections were also associated with a significantly greater net loss $(P \leq .01)$, and underlying disease significantly impacted readmissions $(P=.03)$. The number of prior admissions, hospital characteristics, and payer type were also found to significantly impact measured outcomes.

Conclusion: Carbapenem-nonsusceptible respiratory infections are associated with a considerable clinical and economic burden. The impact of hospital-onset infections on both clinical and economic outcomes highlights the continued need for action on this modifiable risk factor through antimicrobial stewardship and optimal therapy, thereby reducing the burden in this patient population.

Keywords: carbapenem-nonsusceptible, cost, gram-negative, mortality, respiratory infection

\section{Plain Language Summary}

Gram-negative bacteria like Klebsiella and Pseudomonas spp. are a major cause of hospitalacquired bacterial pneumonia and ventilator-associated bacterial pneumonia. Increasing resistance to antibacterial agents is a global health issue that complicates treatment and increases the burden to both patients and health care systems. Infections that cannot be effectively treated by carbapenem antibacterial agents (nonsusceptible) are associated with poor patient outcomes compared with infections caused by carbapenem-susceptible isolates. In this study, we 
evaluated patient, infection, and hospital characteristics to identify variables that may lead to worse outcomes in patients with carbapenem-nonsusceptible respiratory infections. We analyzed data from almost 1500 confirmed gram-negative pneumonia infections over a 2-year period. Patients who required mechanical ventilation, were admitted to the intensive care unit, or who had hospitalacquired infections had the highest risk of mortality. Risk of readmission increased in patients who had previous hospitalizations within the past 90 days. Hospital-onset infection was also the most significant predictor of both length of hospital stay and increased hospital costs. The only modifiable risk factor identified in this study was hospital-onset infection. These findings highlight the importance of infection control measures in lessening patient and economic burden associated with carbapenem-nonsusceptible gram-negative pneumonia.

\section{Introduction}

Respiratory infections, including pneumonia, are one of the leading causes of mortality in the United States (US). ${ }^{1}$ Although hospitalizations and deaths due to pneumonia appear to be declining, ${ }^{1,2}$ a study of hospitalization rates from 2002 through 2011 found significant increases in infections attributed to the gram-negative pathogens Klebsiella and Pseudomonas spp., with increases of $35 \%$ and $23 \%$, respectively $(P<.001) .^{3}$ In the hospital setting, gram-negative bacteria are implicated as the primary causative pathogens in most hospital-acquired or ventilatorassociated bacterial pneumonia patients. ${ }^{4-6}$

Numerous antibiotic classes are currently available for the treatment of gram-negative infections; however, the increasing incidence of antibiotic resistance has become a global health problem. ${ }^{7}$ Antimicrobial surveillance programs of respiratory isolates have documented decreased susceptibility to a variety of antibiotics, including carbapenems. ${ }^{5,8}$ Although fewer than $10 \%$ of respiratory infections in the US are carbapenemnonsusceptible, the incidence is increasing, especially in hospital-acquired infections. ${ }^{8}$ For the gram-negative pathogens Acinetobacter baumannii and Pseudomonas aeruginosa, $50 \%$ and $19 \%$ of respiratory infections tested carbapenemnonsusceptible, respectively. ${ }^{9}$ In general, carbapenem nonsusceptibility is associated with worsened patient outcomes, including prolonged hospital length of stay (LOS), ${ }^{9,10}$ increased costs, ${ }^{11}$ and increased risk of mortality, ${ }^{9,10}$ compared with carbapenem-susceptible pathogens.

Limited data are available on factors that contribute to the clinical and economic burden associated with carbapenem-nonsusceptible gram-negative infections, with existing data typically limited to one setting of care or patient population. This large, retrospective, multicenter analysis examined patient- and hospital-level predictor variables that led to worse outcomes in patients with carbapenemnonsusceptible respiratory infections using data from 78 US acute care hospitals.

\section{Materials and Methods Data Source}

We used electronically captured microbiologic and administrative data from the BD Insights Research Database (Becton, Dickinson and Company; Franklin Lakes, NJ, USA). ${ }^{12-14}$ The dataset for this study included patient data (ie, age, sex, clinical severity, and number of hospitalizations), microbiologic data (ie, specimen collection time, source, and culture results), census data (ie, care location), and postdischarge administrative data (ie, principal diagnosis, discharge disposition, payer, hospital LOS, total cost, and payment received by the hospital). The study dataset was a deidentified and limited retrospective dataset exempted from patient consent by the New England Institutional Review Board (Wellesley, MA, USA). The study was conducted in compliance with the Health Insurance Portability and Accountability Act of 1996. All procedures followed were in accordance with the ethical standards of the Helsinki Declaration.

\section{Study Population}

This study included nonduplicate (the first isolate of any species obtained from a patient per 30-day period) respiratory isolates from consecutive adult inpatients who were admitted from January 1, 2013, through September 30, 2015, and had culture-confirmed gram-negative pathogens, from a respiratory source, that were nonsusceptible to carbapenem.

\section{Definition of Carbapenem Nonsusceptibility}

Based on the Centers for Disease Control and Prevention National Healthcare Safety Network definition, ${ }^{15}$ carbapenem-nonsusceptibility was defined as isolates that tested "resistant" or "intermediate" to imipenem or meropenem for $P$. aeruginosa or A. baumannii, or to imipenem, meropenem, doripenem, or ertapenem for Enterobacteriaceae (Escherichia coli, Klebsiella pneumoniae, Proteus mirabilis, Enterobacter cloacae, Enterobacter aerogenes, Serratia marcescens, Citrobacter freundii, or Morganella morganii). Each hospital's interpretation of the results reported in the laboratory information system dictated the 
classification of isolates as "resistant" or "intermediate." Nonduplicate, gram-negative respiratory isolates were classified as community- or hospital-onset based on the specimen collection time ( $<3$ vs $\geq 3$ days from admission, respectively).

\section{Outcomes}

In-hospital mortality, 30-day readmission, LOS, hospital total cost, and net gain/loss (total cost minus payment received) per patient were derived from postdischarge administrative data within the administrative database and the hospital financial database.

\section{Statistical Analysis}

A univariate analysis was conducted to examine the associations between each potential predictor and each outcome measure. The potential predictor variables included age, sex, location of onset (hospital or community), organism, payer, principal diagnosis-based clinical classification software disease category as a measure of underlying clinical conditions (circulatory system diseases included cerebrovascular diseases, diseases of the heart, and diseases of arteries and arterioles as per the definitions set forth in the Agency for Health Research and Quality's Clinical Classification Software), ${ }^{16}$ mechanical ventilation or intensive care unit (ICU) admission status, number of hospital admissions in the 90 days before the index hospitalization, and hospital characteristics (teaching status, number of beds, and geographic location). We also included an aggregated measure of clinical severity using a published Acute Laboratory Risk of Mortality Score (ALaRMS). ${ }^{17}$ The ALaRMS uses patient demographics and 24 numeric laboratory test results to score the probability of inhospital mortality. The laboratory results include serum chemistry (albumin, aspartate transaminase, alkaline phosphatase, blood urea nitrogen, calcium, creatinine, glucose, potassium, sodium, and total bilirubin); hematology and coagulation parameters (bands, hemoglobin, partial thromboplastin time, prothrombin time international normalized ratio, platelets, and white blood cell count); arterial blood gas (partial pressure of carbon dioxide, partial pressure of oxygen, and $\mathrm{pH}$ value); and cardiac markers (brain natriuretic peptide, creatine phosphokinase MB, pro-brain natriuretic peptide, and troponin I or troponin T).

We developed 5 multivariable regression models, one for each outcome measure, using the Statistical Analysis Software (SAS) GLM selection procedure to generate a set of significant covariates and then fit the generalized linear mixed model
(GLMM) based on the significance of covariates and goodness-of-fit model statistics. We chose to use the GLMM approach because it accounts for variations among hospitals by modeling the hospital as a random effect. Specifically, the 2 binary outcomes (mortality and readmission) were modeled using random intercept logistic regression models, and the continuous outcome measures were modeled using GLMM with appropriate link functions such as log-normal or gamma distributions to handle right-skewed data (LOS, total cost). Reference groups were automatically assigned by SAS and were typically the largest single group. The results were converted back to their original scale (ie, days, US dollars) of measurement by using the ILINK option in the SAS GLIMMIX procedure. All analyses were conducted using SAS version 9.4 (SAS Institute, Inc.; Cary, NC, USA).

\section{Results}

\section{Patient Characteristics}

A total of 1488 carbapenem-nonsusceptible respiratory patients were identified and included in the analysis. Most patients were male (56.4\%), and $35.9 \%$ of patients were $\geq 65$ years of age (Supplementary Table 1). Medicare was the payer for almost one-half of patients (49.1\%), followed closely by private/other insurance (41.7\%), with only $9.2 \%$ covered by Medicaid. Almost two-thirds of patients had community-onset infections (63.8\%). P. aeruginosa (71.8\%) was the most common pathogen, followed by polymicrobial infections $(\geq 1$ pathogen isolated from the respiratory source; 13.2\%), Enterobacteriaceae (10.7\%), and A. baumannii (4.4\%). The most common polymicrobial combinations were $K$. pneumoniae AND $P$. aeruginosa $(16 \%$ of all patients with polymicrobial infection), followed by $A$. baumannii AND $P$. aeruginosa (14\%), and E. coli AND P. aeruginosa $(14 \%)$.

\section{Univariate Analysis of Associated Outcomes}

For the overall population, the mortality rate was $13.7 \%$ $(\mathrm{n}=204 / 1488)$ and the readmission rate was $20.6 \%(\mathrm{n}=265 /$ 1284 live discharges). The mean (standard deviation [SD]) LOS was 20 (27) days, mean (SD) total cost per patient in US dollars was $\$ 54,158(\$ 98,312)$, and the mean (SD) net gain/loss per patient was $-\$ 139(\$ 92,329$; a loss of $\$ 139)$.

\section{Multivariable Analysis of Associated Outcomes}

In the multivariable analysis, predictor variables significantly associated with the greatest increase in risk of 
mortality included ALaRMS in the fourth quartile (odds ratio [OR], 3.29; 95\% confidence interval [CI], 2.06-5.25; $P<.001)$, mechanical ventilation or ICU admission status (OR, 2.92; 95\% CI, 1.75-4.86; $P<.001$ ), ages 55 to 64 years (OR, 2.79; 95\% CI, 1.09-7.10; $P=.03$ ), hospitalonset infection (OR, 2.35; 95\% CI, 1.60-3.45; $P<.001$ ), and the hospital located in the South geographic region (OR, 2.30; 95\% CI, 1.23-4.30; $P=.01$ ) (Figure 1). Other predictor variables significantly associated with increased risk included infectious and parasitic diseases as the principal diagnosis, polymicrobial organisms as the causative pathogens, and ALaRMS in the second and third quartiles.

For 30-day readmission, the predictor variable significantly associated with the greatest increase of risk was more than one hospital admission in the 90 days before the index hospitalization (OR, 3.71; 95\% CI, 2.19-6.28; $P<.001$ ) (Figure 2). Other significant predictors associated with increased risk included diseases of the circulatory system (OR, 2.55; 95\% CI, 1.08-6.02; $P=.03$ ) and infectious and parasitic diseases (OR, 1.90; 95\% CI, 1.07-3.39; $P=.03)$ as the principal diagnosis. Two predictor variables were significantly associated with a decreased risk of 30day readmission: no hospital admissions in the 90 days before the index hospitalization (OR, 0.004; 95\% CI, 0.$001-0.19 ; P<.001)$ and teaching hospital status (OR, 0.62; 95\% CI, 0.43-0.89; $P=.01$ )

Predictor variables significantly associated with the greatest increase in LOS included hospital-onset infections (estimated LOS of 23.3 days [95\% CI, 20.4-26.7; $P<.001]$ vs 9.5 days [95\% CI, 8.4-10.8] for those with communityonset infections), polymicrobial infection (estimated LOS of 20.3 days [95\% CI, 17.1-24.1; $P<.001]$ vs 15.0 days [95\% CI, 13.7-16.3] for $P$. aeruginosa infection), mechanical ventilation or ICU admission status (estimated LOS of 17.4 days [95\% CI, 15.2-20.0; $P<.001]$ vs 12.7 days [95\% CI, 11.3-14.4] for those without mechanical ventilation/ICU admission), and South geographic location (estimated LOS of 17.0 days [95\% CI, 15.2-19.1; $P<.001$ ] vs 12.6 days [95\% CI, 11.3-14.0] for Midwest geographic location) (Figure 3). Other predictors associated with significantly increased LOS included the principal diagnosis (diseases of the circulatory system, endocrine/nutritional/
Predictor Variables:

\section{Demographics}

Severity

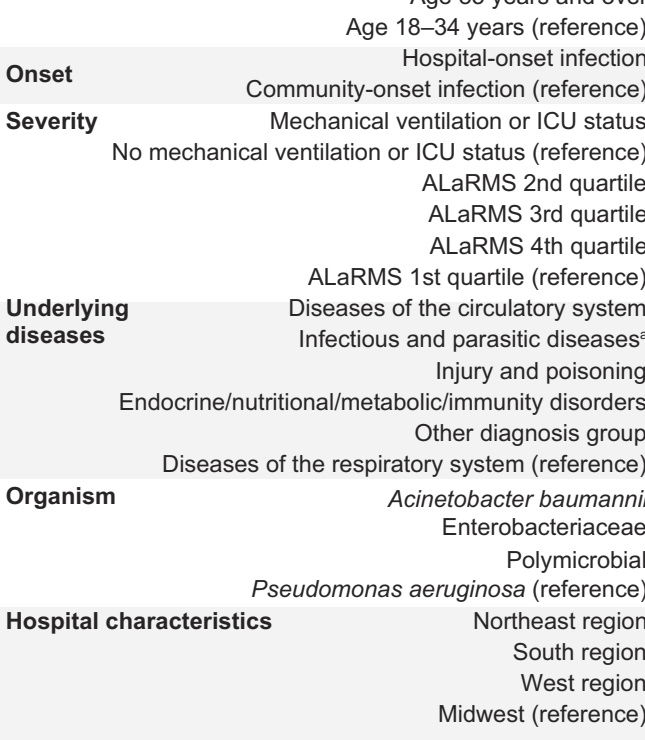

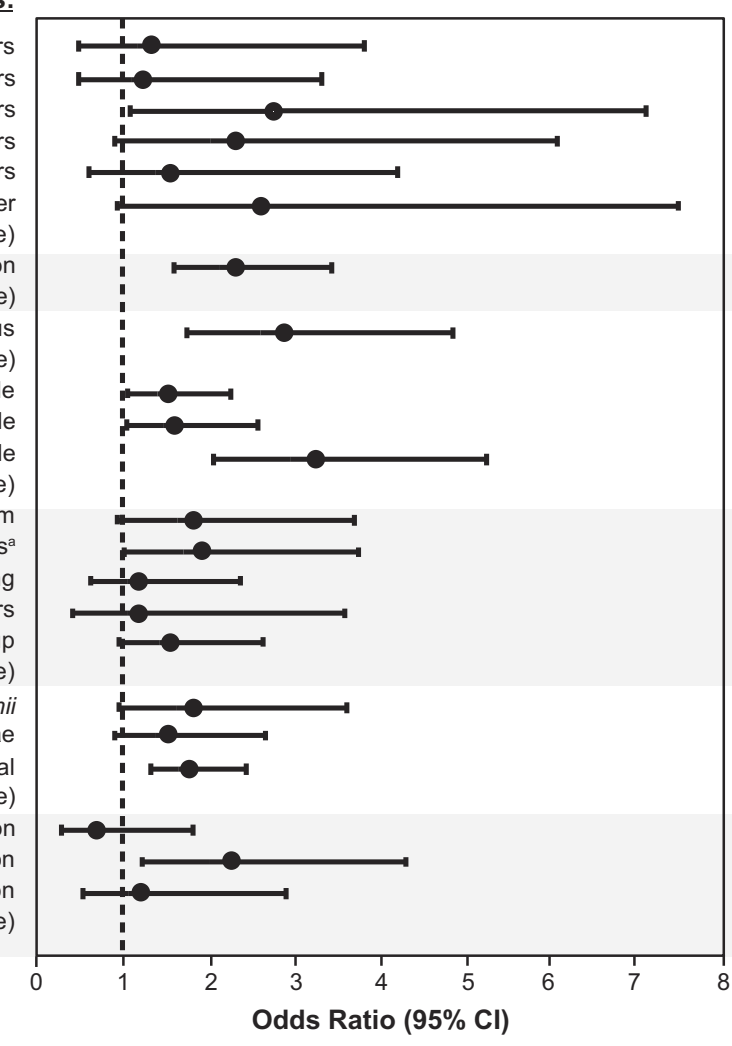

\section{Odds Ratio $(95 \% \mathrm{Cl})$}

$1.37(0.49,3.81) ; P=.55$

$1.28(0.49,3.34) ; P=.62$

2.79 (1.09, 7.10); $P=.03$

$2.35(0.91,6.07) ; P=.08$

$1.60(0.61,4.22) ; P=.34$

$2.65(0.94,7.47) ; P=.06$

2.35 (1.60, 3.45); $P<.001$

$2.92(1.75,4.86) ; P<.001$

$1.56(1.06,2.28) ; P=.02$

$1.65(1.05,2.59) ; P=.03$

3.29 (2.06, 5.25); $P<.001$

$1.87(0.94,3.71) ; P=.07$

$1.96(1.02,3.75) ; P=.04$

$1.22(0.63,2.39) ; P=.56$

$1.22(0.42,3.59) ; P=.71$

$1.60(0.96,2.64) ; P=.07$

$1.86(0.96,3.62) ; P=.07$

$1.56(0.91,2.68) ; P=.11$

$1.81(1.33,2.46) ; P<.001$

$0.73(0.29,1.83) ; P=.50$

$2.30(1.23,4.30) ; P=.01$

$1.25(0.54,2.92) ; P=.60$

Figure I Multivariable analysis: mortality model.

Notes: Dashed line denotes unity (the line of no effect). Odds ratios for predictor variables are relative to a reference population for each variable grouping. Statistical significance $(P<.05)$ is indicated in bold text. Infectious diseases were predominantly septicemia.

Abbreviations: ALaRMS, Acute Laboratory Risk of Mortality Score; $\mathrm{Cl}$, confidence interval; ICU, intensive care unit; $P$, $\mathrm{P}$ value. 


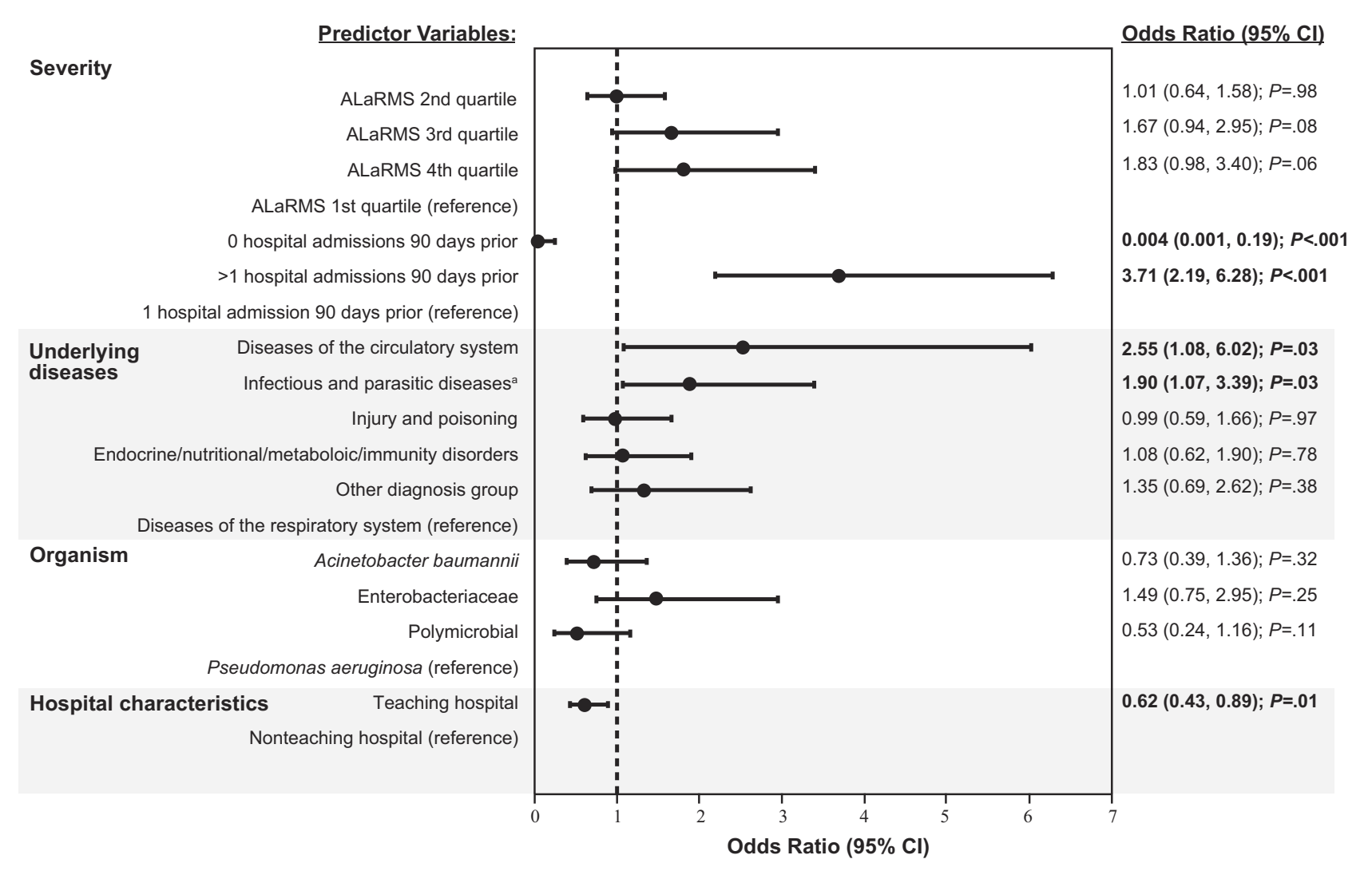

Figure 2 Multivariable analysis: 30-day readmission model.

Notes: Dashed line denotes unity (the line of no effect). Odds ratios for predictor variables are relative to a reference population for each variable grouping. Statistical

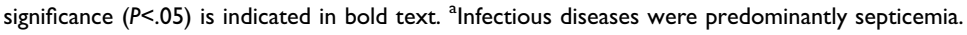

Abbreviations: ALaRMS, Acute Laboratory Risk of Mortality Score; $\mathrm{Cl}$, confidence interval; $P, \mathrm{p}$ value.

metabolic/immunity disorders, and injury and poisoning), ALaRMS in the fourth quartile, hospital size of $>300$ beds, and West geographic location. Age groups of 35-44 and $>85$ years and $A$. baumannii infection were predictor variables significantly associated with decreased LOS.

Predictor variables significantly associated with the highest total cost were hospital-onset infections $(\$ 73,921$ vs $\$ 24,691$ for community-onset infections; $P<.001$ ), diseases of the circulatory system as the principal diagnosis $(\$ 60,439$ vs $\$ 34,384$ for respiratory diseases; $P<.001$ ), polymicrobial infection ( $\$ 60,337$ vs $\$ 41,701$ for $P$. aeruginosa; $P<.001)$, and mechanical ventilation or ICU admission status $(\$ 59,429$ vs $\$ 30,712$ without mechanical ventilation/ICU admission; $P<.001$ ) (Figure 4). Other independent predictors significantly associated with higher total cost included teaching hospital status, West geographic location, ALaRMS in the third or fourth quartiles, and principal diagnosis of injury or poisoning.

For net loss to the hospital, although the overall loss appeared reasonably low at $-\$ 139$, the magnitude of gain/ loss was greater when individual variables were analyzed.
Predictor variables significantly associated with greater losses included Medicaid as the payer ( $\$ 47,553$ vs $\$ 14,653$ gain for private insurance/other payer; $P=.02$ ), more than one hospital admission in the 90 days before the index hospitalization ( $-\$ 25,279$ vs $-\$ 10,900$ for 1 admission; $P<.01)$, hospital-onset infections $(-\$ 20,460$ vs $-\$ 11,031$ for community-onset infections; $P=.01$ ), and Medicare as the payer ( $\$ 14,337$ vs $\$ 14,653$ gain for private insurance/other payer; $P=.02$ ) (Figure 5).

\section{Discussion}

The clinical and economic burden associated with respiratory infections is relatively well documented; however, few studies have focused on the identification of contributory factors, particularly for carbapenem-nonsusceptible gram-negative infections. In this study, we conducted univariate and multivariate analyses to identify patient- and hospital-level predictor variables associated with increased mortality, 30-day readmission, LOS, total cost of care, and net gain or loss. Our findings show several predictors with a significant effect on multiple outcomes, including those 


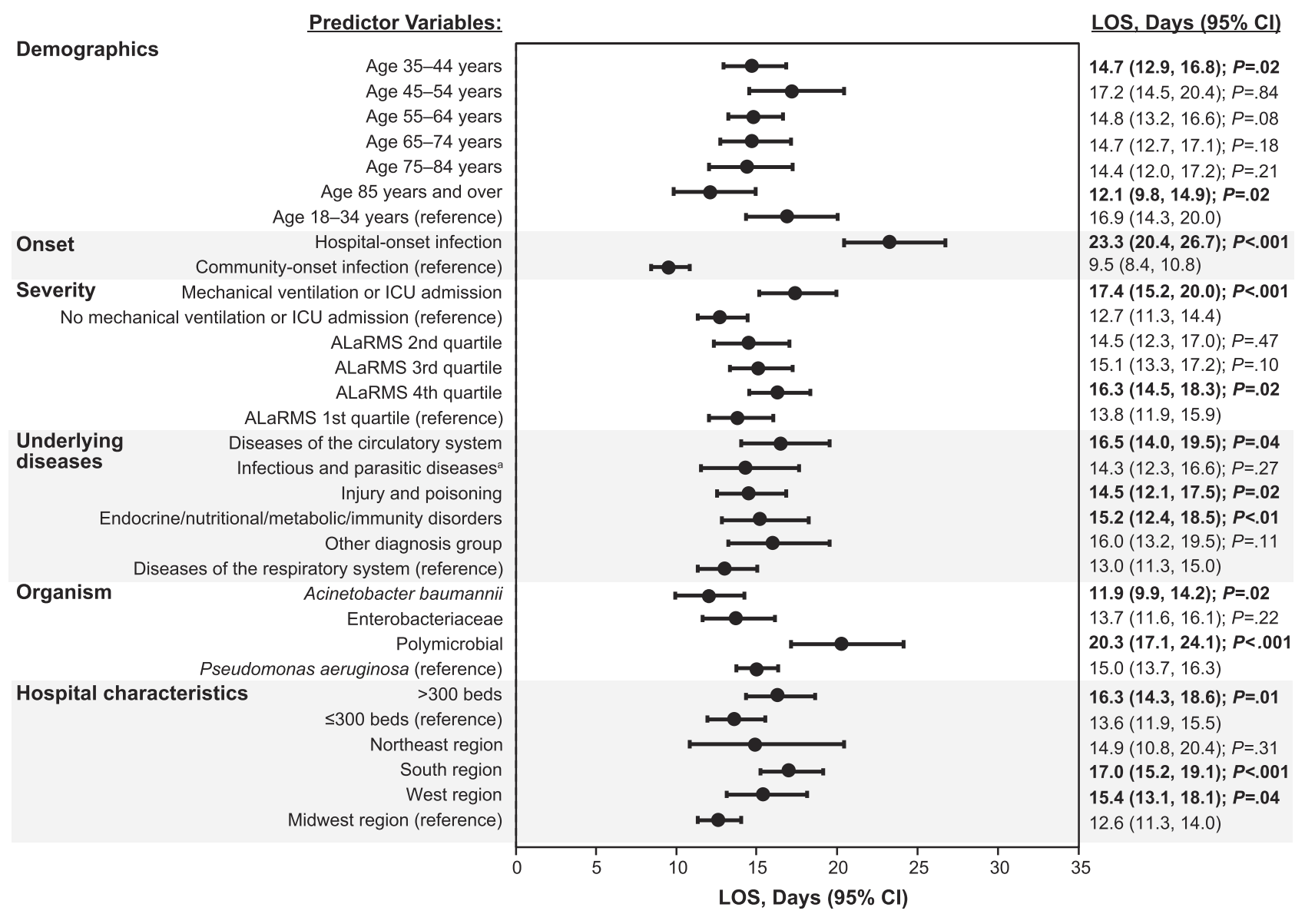

Figure 3 Multivariable analysis: LOS model.

Notes: ${ }^{a}$ Infectious diseases were predominantly septicemia. Statistical significance $(P<.05)$ is indicated in bold text.

Abbreviations: ALaRMS, Acute Laboratory Risk of Mortality Score; Cl, confidence interval; ICU, intensive care unit; LOS, length of stay; $P$, $\mathrm{P}$ value.

relating to the onset setting, clinical severity, infecting organism, and underlying disease(s).

Hospital-onset infection was the predictor variable with a significant impact on the most outcome measures evaluated (4 of 5) and corresponded with approximately double the risk of mortality, an increased LOS of $\sim 14$ days, $\sim \$ 49,000$ increased total cost, and an $\sim \$ 9000$ additional loss per patient compared with community-onset infections. Our findings of increased clinical and economic burden for hospital-onset infections within this relatively large sample are consistent with the published literature for healthcare-associated pneumonia in general, particularly in patients with carbapenemnonsusceptible gram-negative respiratory infections. ${ }^{9,11,18,19}$ The overall mortality rate, LOS, and total cost observed for patients with hospital-onset infection were among the highest observed in the univariate analysis of predictor variables and therefore reinforce the need for hospitals to align with recent action plans to improve infection control and prevention in the hospital setting. ${ }^{20,21}$
ALaRMS in the fourth quartile was significantly associated with an $\sim 3$-fold increased mortality risk, increased LOS of $\sim 3$ days, and $\sim \$ 17,000$ increased total cost compared with values in the first quartile. The significant association between disease severity, as measured by objective laboratory test results, and worse clinical and economic outcomes is also consistent with the literature. Previous studies have observed a significant association between higher scores for the Charlson Comorbidity Index, quick Sequential Organ Failure Assessment, and Acute Physiology and Chronic Health Evaluation (APACHE) II and increased mortality risk or mortality rates in patients hospitalized with pneumonia; ${ }^{22-25}$ however, few studies have examined the association of these prognostic tools with economic outcomes and, to our knowledge, no other studies have estimated the net loss per patient for each risk factor for the study population. A retrospective study by Lye et al identified a positive association of higher APACHE II scores and higher LOS among patients with healthcare-associated and nosocomial gram-negative bacteremia. ${ }^{26}$ Although the higher observed LOS could be 
Predictor Variables:

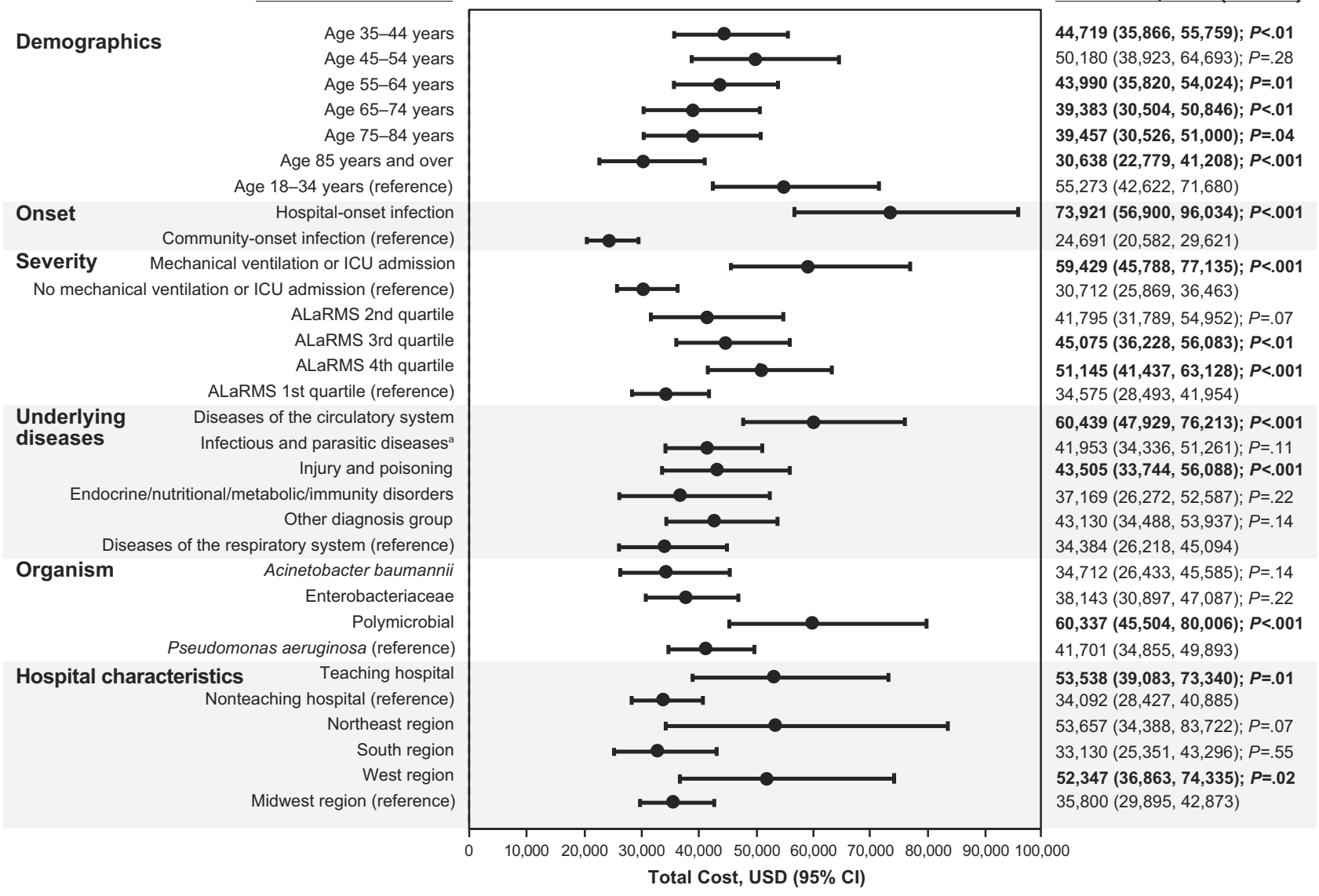

Figure 4 Multivariable analysis: hospital total cost model.

Notes: ${ }^{a}$ Infectious diseases were predominantly septicemia. Statistical significance $(P<.05)$ is indicated in bold text.

Abbreviations: ALaRMS, Acute Laboratory Risk of Mortality Score; Cl, confidence interval; ICU, intensive care unit; $P$, P value; USD, United States dollars.

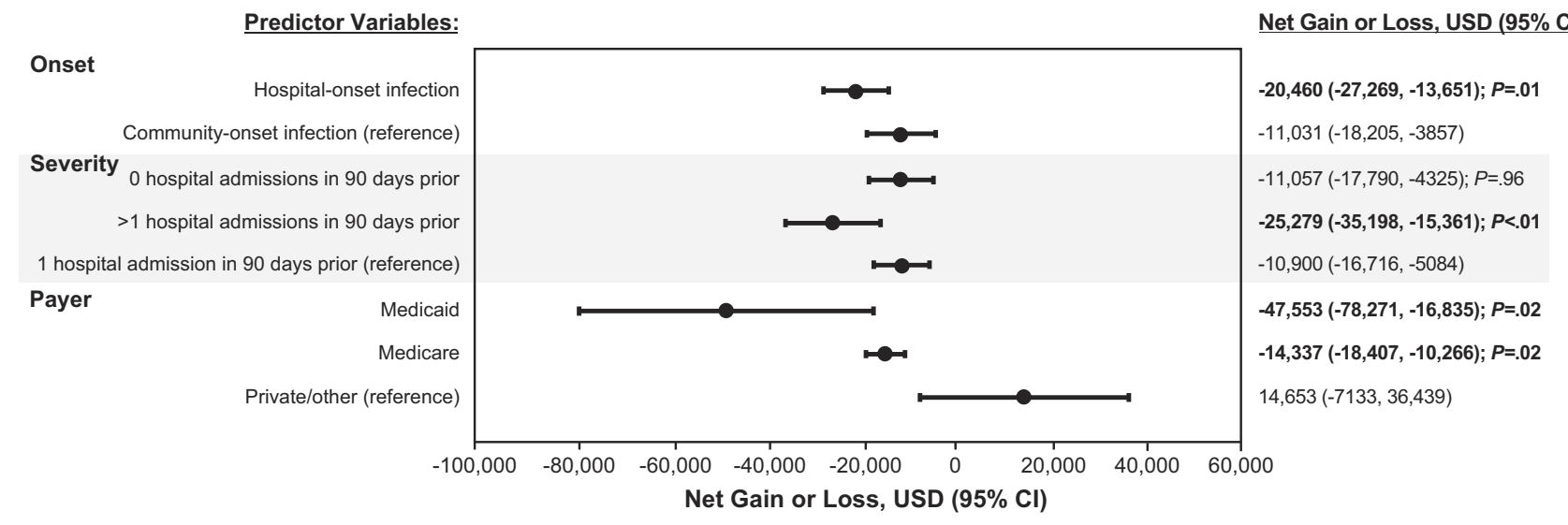

Figure 5 Multivariable analysis: net gain or loss model.

Notes: Statistical significance $(P<.05)$ is indicated in bold text.

Abbreviations: $\mathrm{Cl}$, confidence interval; $P$, $\mathrm{P}$ value; USD, United States dollars.

expected to result in increased total cost, the authors did not report directly on costs.

Patients hospitalized with carbapenem-resistant gramnegative infections are much more likely to be treated in the ICU or require mechanical ventilation. ${ }^{27,28}$ In our study, positive mechanical ventilation/ICU admission status was significantly associated with an $\sim 3$-fold increased mortality risk, increased LOS of $\sim 5$ days, and $\sim \$ 29,000$ increased 
total cost compared with negative mechanical ventilation/ ICU status. Mechanical ventilation and ICU admission status are well-known predictors of increased mortality in patients with respiratory infections regardless of pathogen or presence of carbapenem resistance. ${ }^{29}$ In terms of LOS and economic burden, 2 retrospective studies of patients admitted to the ICU who developed ventilator-associated pneumonia (VAP) observed significantly increased LOS ( $\sim 12$ to 13 days) and increased hospital charges per patient $(\sim 40,000)$ compared with patients without VAP. ${ }^{30,31}$ However, these studies did not limit the population of interest to those with laboratory-confirmed carbapenemnonsusceptible infections. Nonetheless, the overall body of evidence supports the inclusion of mechanical ventilation and ICU status in risk assessment algorithms, highlighting the need to ensure that high-risk patients are promptly and adequately treated upon ICU admission and that the mechanical ventilation procedure is optimally managed to prevent VAP.

Polymicrobial infections occurred in $\sim 13 \%$ of patients in our study population and were significantly associated with an $\sim 2$-fold higher mortality risk, increased LOS of $\sim 5$ days, and $\sim \$ 19,000$ increased total cost compared with the reference population of $P$. aeruginosa infections. Polymicrobial bacterial infections are estimated to comprise almost onethird of all patients of ventilator-associated tracheobronchitis, a precursor to VAP. $^{29}$ Polymicrobial respiratory infections are also common in patients with other comorbidities such as chronic obstructive pulmonary disease, cystic fibrosis, and cancer. $^{32-34}$ Although several observational studies have investigated the burden associated with tracheobronchitis and VAP, these studies considered monomicrobial and polymicrobial infections together, so it is difficult to make direct comparisons to our findings on burden. ${ }^{33,35}$

A variety of predictor variables in the underlying diseases category were also significantly associated with worse burden. Diseases of the circulatory system were significantly associated with increased risk of 30-day readmission ( $\sim 3$-fold higher), increased LOS ( $\sim 4$ additional days), and increased total cost ( $\$ 26,000$ higher) compared with the reference population of diseases of the respiratory system. The predictor variable of infectious and parasitic diseases, predominately sepsis pneumonia patients, was significantly associated with an increased risk of mortality and 30-day readmission (both $\sim 2$-fold higher), and injury and poisoning was significantly associated with increased LOS ( $\sim 2$ days) and increased total cost ( $\$ 9000$ higher). Carbapenem-nonsusceptible, gram- negative respiratory infections are serious and complicated infections to treat and manage, and our observations highlight that existing underlying diseases compound the burden to the patient and increase the need for hospital resources.

The age groupings that were analyzed as predictor variables were not significantly or consistently associated with worse outcomes or increased burden. For example, 55-64 years was the only age group significantly associated with an increased mortality risk (OR, 2.79; $P=.03$ ) while the highest age group ( $>85$ years) was not (OR, $2.65 ; P=.06)$. In addition, hospital LOS was significantly decreased rather than increased for age $>85$ years, and total costs were significantly decreased for all age groups, relative to the reference population of age 18-34 years. The lack of a consistent independent effect of age might be due to the correlations of covariates in the model with other variables that already accounted for the effect of advanced age. For example, older age was positively correlated with greater disease severity as measured by ALaRMS, ICU status, mechanical ventilation, and exposure time (length of stay prior to the onset of infection; correlation coefficients ranged from 0.20 to 0.60 , all $P<.0001$ ).

This study is strengthened by inclusion of data from a large number of patients with laboratory-confirmed, nonduplicate, carbapenem-nonsusceptible respiratory isolates obtained from 78 US acute care hospitals. The inclusion of hospital cost data (as opposed to claims data) for the calculation of net gain/loss provides additional value from the providers' perspective. Limitations include the retrospective nature of the study, which can be subject to potential biases and confounding. In addition, despite inclusion of data from 78 hospitals, the study population is not necessarily representative of patients from across the US. During the period of our analysis (2013-2015), the CDC definition of CRE transitioned. For our study, we utilized the older definition that included strains with either resistant [R] or intermediate [I] susceptibilities to imipenem, meropenem, or doripenem; however, the newer definition only includes Enterobacteriaceae that test $\mathrm{R}$ to imipenem, meropenem, doripenem, or ertapenem. ${ }^{15}$ According to data presented by Weiner and colleagues, very few isolates were categorized with I susceptibility and they comprised only a small proportion of the total isolates in their study. ${ }^{15}$ Therefore, we do not anticipate that use of the updated CRE definition in our analysis would appreciably impact the predictive models. Additionally, the definition of carbapenem nonsusceptibility that we used for Enterobacteriaceae includes $P$. 
mirabilis and M. morganii, which have intrinsically elevated MICs to imipenem. However, only $1.8 \%$ of the study population had $P$. mirabilis and only $0.9 \%$ of the study population had $M$. morganii. Therefore, the inclusion of these patients is unlikely to have a significant impact on the model fit.

\section{Conclusion}

These data confirm that carbapenem-nonsusceptible gramnegative respiratory infections are associated with a considerable clinical and economic burden. Patients with certain key characteristics (hospital-onset infection, greater clinical severity, need for mechanical ventilation or ICU admission, polymicrobial infection, and certain major underlying diseases) may be at increased risk of mortality, longer LOS, and higher costs. Hospital-onset infection was associated with the most significantly impacted outcomes (ie, mortality, LOS, total cost, and net loss), underscoring the need for continued efforts in hospital infection prevention and antimicrobial stewardship to ensure optimal coverage of pathogens. Given that hospitalonset infection was the only modifiable risk factor identified in this study, a focused effort on prevention represents the best opportunity to reduce the clinical and economic burden associated with these infections.

\section{Abbreviations}

ALaRMS, Acute Laboratory Risk of Mortality Score; APACHE, Acute Physiology and Chronic Health Evaluation; CI, confidence interval; GLMM, generalized linear mixed model; ICU, intensive care unit; LOS, length of stay; OR, odds ratio; $\mathrm{SD}$, standard deviation; VAP, ventilator-associated pneumonia.

\section{Data Sharing Statement}

MSD's data sharing policy, including restrictions, is available at http://engagezone.msd.com/ds_documentation.php. Requests for access to the study data can be submitted through the EngageZone site or via email to dataaccess@merck.com.

\section{Acknowledgments}

The authors thank Dr. Richard Johannes, for his dedicated contributions in clinical and scientific insight on the early stage of this study, and Vikas Gupta, PharmD, for his clinical insight. Medical writing and/or editorial assistance provided by Amy Agbonbhase, $\mathrm{PhD}$, and Robert Schupp, PharmD, of The Lockwood Group, Stamford, CT, USA, was funded by MSD.

\section{Author Contributions}

All authors are responsible for the work described in this paper. All authors made substantial contributions to conception and design, acquisition of data, or analysis and interpretation of data; took part in drafting the article or revising it critically for important intellectual content; gave final approval of the version to be published; and agree to be accountable for all aspects of the work in ensuring that questions related to the accuracy or integrity of any part of the work are appropriately investigated and resolved. Study concept and design: EM, AHS, GY, and YPT. Data analysis and interpretation: EM, AHS, GY, LV, and YPT. Drafting manuscript: EM and YPT. Critical revision of manuscript: EM, AHS, GY, LV, and YPT. Study supervision: YPT. Final approval of manuscript: EM, AHS, GY, LV, and YPT. YPT has access to all the data for the current study, and she takes responsibility for data accuracy and integrity.

\section{Funding}

Funding for this research was provided by Merck Sharp \& Dohme Corp., a subsidiary of Merck \& Co., Inc., Kenilworth, NJ, USA (MSD).

\section{Disclosure}

EM is an employee of MSD and may own stock and/or hold stock options in Merck \& Co., Inc., Kenilworth, NJ, USA. AHS was previously employed by MSD and may own stock and/or hold stock options in Merck \& Co., Inc., Kenilworth, NJ, USA. GY, LV, and YPT are full-time employees of Becton, Dickinson and Company, Franklin Lakes, NJ, USA. The authors report no other conflicts of interest in this work.

\section{References}

1. Kochanek KD, Murphy SL, Xu J, Tejada-Vera B Deaths: final data for 2014. 2016. Available from: https://www.cdc.gov/nchs/data/nvsr/ nvsr65/nvsr65_04.pdf. Accessed October 2, 2017.

2. Pfuntner A, Wier LM, Stocks C Statistical brief \#162: most frequent conditions in U.S. hospitals, 2011. 2013. Available from: https://www. ncbi.nlm.nih.gov/books/NBK169248/pdf/Bookshelf_NBK169248.pdf. Accessed October 2, 2017.

3. Wuerth BA, Bonnewell JP, Wiemken TL, Arnold FW. Trends in pneumonia mortality rates and hospitalizations by organism, United States, 2002-2011(1). Emerg Infect Dis. 2016;22(9):1624-1627. doi:10.3201/eid2209.150680

4. Djordjevic ZM, Folic MM, Jankovic SM. Distribution and antibiotic susceptibility of pathogens isolated from adults with hospital-acquired and ventilator-associated pneumonia in intensive care unit. $J$ Infect Public Health. 2017;10(6):740-744. doi:10.1016/j.jiph.2016.11.016 
5. Jones RN. Microbial etiologies of hospital-acquired bacterial pneumonia and ventilator-associated bacterial pneumonia. Clin Infect Dis. 2010;51(Suppl 1):S81-S87. doi:10.1086/653053

6. Sader HS, Farrell DJ, Flamm RK, Jones RN. Antimicrobial susceptibility of gram-negative organisms isolated from patients hospitalised with pneumonia in US and European hospitals: results from the SENTRY Antimicrobial Surveillance Program, 2009-2012. Int J Antimicrob Agents. 2014;43(4):328-334. doi:10.1016/j.ijantimicag.2014.01.007

7. World Health Organization. Antimicrobial resistance global report on surveillance. 2014. Available from: http://apps.who.int/iris/bitstream/ 10665/112642/1/9789241564748_eng.pdf?ua=1. Accessed October 2, 2017.

8. Thaden JT, Lewis SS, Hazen KC, et al. Rising rates of carbapenemresistant Enterobacteriaceae in community hospitals: a mixed-methods review of epidemiology and microbiology practices in a network of community hospitals in the southeastern United States. Infect Control Hosp Epidemiol. 2014;35(8):978-983. doi:10.1086/677157

9. Cai B, Echols R, Magee G, et al. Prevalence of carbapenem-resistant gram-negative infections in the United States predominated by Acinetobacter baumannii and Pseudomonas aeruginosa. Open Forum Infect Dis. 2017;4(3):ofx176. doi:10.1093/ofid/ofx176

10. Muggeo A, Guillard T, Barbe C, et al. Factors associated with carriage of carbapenem-non-susceptible Enterobacteriaceae in North-Eastern France and outcomes of infected patients. J Antimicrob Chemother. 2017;72 (5):1496-1501. doi:10.1093/jac/dkw590

11. Bartsch SM, McKinnell JA, Mueller LE, et al. Potential economic burden of carbapenem-resistant Enterobacteriaceae (CRE) in the United States. Clin Microbiol Infect. 2017;23(1):48.e49-48.e16. doi:10.1016/j.cmi.2016.09.003

12. Tabak YP, Zilberberg MD, Johannes RS, Sun X, McDonald LC. Attributable burden of hospital-onset Clostridium difficile infection: a propensity score matching study. Infect Control Hosp Epidemiol. 2013;34(6):588-596. doi:10.1086/670621

13. Zilberberg MD, Tabak YP, Sievert DM, et al. Using electronic health information to risk-stratify rates of Clostridium difficile infection in US hospitals. Infect Control Hosp Epidemiol. 2011;32(7):649-655. doi: $10.1086 / 660360$

14. Gross AE, Johannes RS, Gupta V, Tabak YP, Srinivasan A, Bleasdale SC. The effect of a piperacillin/tazobactam shortage on antimicrobial prescribing and Clostridium difficile risk in 88 US medical centers. Clin Infect Dis. 2017;65(4):613-618. doi:10.1093/cid/cix379

15. Weiner LM, Webb AK, Limbago B, et al. Antimicrobial-resistant pathogens associated with healthcare-associated infections: summary of data reported to the National Healthcare Safety Network at the Centers for Disease Control and Prevention, 2011-2014. Infect Control Hosp Epidemiol. 2016;37(11):1288-1301. doi:10.1017/ ice.2016.174

16. Agency for Health Care Research and Quality. Clinical Classifications Software (CCS) for ICD-9-CM. 2015. Available from: https://www.hcupus.ahrq.gov/toolssoftware/ccs/ccs.jsp. Accessed June 5, 2019.

17. Tabak YP, Sun X, Nunez CM, Johannes RS. Using electronic health record data to develop inpatient mortality predictive model: Acute Laboratory Risk of Mortality Score (ALaRMS). J Am Med Inform Assoc. 2014;21(3):455-463. doi:10.1136/amiajnl-2013-001790

18. Corrado RE, Lee D, Lucero DE, Varma JK, Vora NM. Burden of adult community-acquired, health-care-associated, hospital-acquired, and ventilator-associated pneumonia: New York City, 2010 to 2014. Chest. 2017;152(5):930-942. doi:10.1016/j.chest.2017.04.162

19. Zimlichman E, Henderson D, Tamir O, et al. Health care-associated infections: a meta-analysis of costs and financial impact on the US health care system. JAMA Intern Med. 2013;173(22):2039-2046. doi:10.1001/jamainternmed.2013.9763
20. Allegranzi B, Kilpatrick C, Storr J, et al. Global infection prevention and control priorities 2018-22: a call for action. Lancet Glob Health. 2017;5(12):E1178-E1180. doi:10.1016/S2214-109X(17)30427-8

21. Office of Disease Prevention and Health Promotion. National Action Plan to Prevent Health Care-Associated Infections: Road Map to Elimination. US Department of Health and Human Services; 2013. Available from: https://health.gov/sites/default/files/2019-09/haiaction-plan-executive-summary.pdf. Accessed June 11, 2019.

22. Bahlis LF, Diogo LP, Kuchenbecker RS, Fuchs SC. Clinical, epidemiological, and etiological profile of inpatients with community-acquired pneumonia in a public hospital in the interior of Brazil. J Bras Pneumol. 2018;44(4):261-266. doi:10.1590/s1806-37562017000000434

23. de Miguel-diez J, Lopez-de-Andres A, Hernandez-Barrera V, et al. Decreasing incidence and mortality among hospitalized patients suffering a ventilator-associated pneumonia: analysis of the Spanish national hospital discharge database from 2010 to 2014. Medicine (Baltimore). 2017;96 (30):e7625. doi:10.1097/MD.0000000000007625

24. Jiang J, Yang J, Jin Y, Cao J, Lu Y. Role of qSOFA in predicting mortality of pneumonia: a systematic review and meta-analysis. Medicine (Baltimore). 2018;97(40):e12634. doi:10.1097/MD.0000000000012634

25. Talbot GH, Das A, Cush S, et al. Evidence-based study design for hospital-acquired bacterial pneumonia and ventilator-associated bacterial pneumonia. J Infect Dis. 2019;219(10):1536-1544. doi:10.1093/infdis/ jiy578

26. Lye DC, Earnest A, Ling ML, et al. The impact of multidrug resistance in healthcare-associated and nosocomial gram-negative bacteraemia on mortality and length of stay: cohort study. Clin Microbiol Infect. 2012;18(5):502-508. doi:10.1111/j.1469-0691.2011.03606.x

27. McCann E, Srinivasan A, DeRyke CA, et al. Carbapenemnonsusceptible gram-negative pathogens in ICU and non-ICU settings in US hospitals in 2017: a multicenter study. Open Forum Infect Dis. 2018;5(10):ofy241. doi:10.1093/ofid/ofy241

28. Zilberberg MD, Nathanson BH, Sulham K, Fan W, Shorr AF. Carbapenem resistance, inappropriate empiric treatment and outcomes among patients hospitalized with Enterobacteriaceae urinary tract infection, pneumonia and sepsis. BMC Infect Dis. 2017;17 (1):279. doi:10.1186/s12879-017-2383-z

29. Kalil AC, Metersky ML, Klompas M, et al. Management of adults with hospital-acquired and ventilator-associated pneumonia: 2016 Clinical Practice Guidelines by the Infectious Diseases Society of America and the American Thoracic Society. Clin Infect Dis. 2016;63 (5):e61-e111. doi:10.1093/cid/ciw353

30. Kollef MH, Hamilton CW, Ernst FR. Economic impact of ventilator-associated pneumonia in a large matched cohort. Infect Control Hosp Epidemiol. 2012;33(3):250-256. doi:10.1086/664049

31. Rello J, Ollendorf DA, Oster G, et al. Epidemiology and outcomes of ventilator-associated pneumonia in a large US database. Chest. 2002;122(6):2115-2121. doi:10.1378/chest.122.6.2115

32. Hardak E, Avivi I, Berkun L, et al. Polymicrobial pulmonary infection in patients with hematological malignancies: prevalence, co-pathogens, course and outcome. Infection. 2016;44(4):491-497. doi:10.1007/s15010-016-0873-3

33. Nseir S, Di Pompeo C, Soubrier S, et al. Outcomes of ventilated COPD patients with nosocomial tracheobronchitis: a case-control study. Infection. 2004;32(4):210-216. doi:10.1007/s15010-004-3167-0

34. Zhao J, Schloss PD, Kalikin LM, et al. Decade-long bacterial community dynamics in cystic fibrosis airways. Proc Natl Acad Sci USA. 2012;109(15):5809-5814. doi:10.1073/pnas.1120577109

35. Nseir S, Di Pompeo C, Soubrier S, et al. Effect of ventilator-associated tracheobronchitis on outcome in patients without chronic respiratory failure: a case-control study. Crit Care. 2005;9(3):R238-R245. doi:10.1186/ $\operatorname{cc} 3508$ 


\section{Publish your work in this journal}

Infection and Drug Resistance is an international, peer-reviewed openaccess journal that focuses on the optimal treatment of infection (bacterial, fungal and viral) and the development and institution of preventive strategies to minimize the development and spread of resistance. The journal is specifically concerned with the epidemiology of antibiotic resistance and the mechanisms of resistance development and diffusion in both hospitals and the community. The manuscript management system is completely online and includes a very quick and fair peerreview system, which is all easy to use. Visit http://www.dovepress.com/ testimonials.php to read real quotes from published authors. 